\title{
Fixed-Time Consensus Tracking for Multi-Agent Systems with High-Order Integrator Dynamics
}

DOI:

10.1109/TAC.2017.2729502

\section{Document Version}

Accepted author manuscript

Link to publication record in Manchester Research Explorer

\section{Citation for published version (APA):}

Zuo, Z., Tian, B., Defoort, M., \& Ding, Z. (2017). Fixed-Time Consensus Tracking for Multi-Agent Systems with High-Order Integrator Dynamics. IEEE Transactions on Automatic Control.

https://doi.org/10.1109/TAC.2017.2729502

\section{Published in:}

IEEE Transactions on Automatic Control

\section{Citing this paper}

Please note that where the full-text provided on Manchester Research Explorer is the Author Accepted Manuscript or Proof version this may differ from the final Published version. If citing, it is advised that you check and use the publisher's definitive version.

\section{General rights}

Copyright and moral rights for the publications made accessible in the Research Explorer are retained by the authors and/or other copyright owners and it is a condition of accessing publications that users recognise and abide by the legal requirements associated with these rights.

\section{Takedown policy}

If you believe that this document breaches copyright please refer to the University of Manchester's Takedown Procedures [http://man.ac.uk/04Y6Bo] or contact uml.scholarlycommunications@manchester.ac.uk providing relevant details, so we can investigate your claim.

\section{OPEN ACCESS}




\title{
Fixed-Time Consensus Tracking for Multi-Agent Systems with High-Order Integrator Dynamics
}

\author{
Zongyu Zuo, Member, IEEE, Bailing Tian, Member, IEEE, Michael Defoort, and Zhengtao Ding, Senior Member, IEEE
}

\begin{abstract}
This paper addresses the fixed-time leader-follower consensus problem for high-order integrator multi-agent systems subject to matched external disturbances. A new cascade control structure, based on a fixedtime distributed observer, is developed to achieve the fixed-time consensus tracking control. A simulation example is included to show the efficacy and the performance of the proposed control structure with respect to different initial conditions.
\end{abstract}

Index Terms-Consensus tracking, Observer, Fixed-time stability, Multiagent system, High-order system

\section{INTRODUCTION}

It is well known that settling time is an important performance specification for a control system, which reflects the convergence rate to any desired state. As an important performance measure also for coordination control of multi-agent systems, fast convergence is always pursued to achieve better performance and robustness, such as hybrid formation flying [1], consensus subject to switching topology [2], [3].

For a linear consensus protocol proposed for single integrator multiagent systems, Olfati-Saber and Murray have demonstrated in [4] that the algebraic connectivity of an interaction graph, i.e., the second smallest eigenvalue of the graph Laplacian, qualified the convergence rate. This motivated Kim and Mesbahi [5] to seek proper interaction topology with larger algebraic connectivity. However, the conventional linear distributed protocols presented in the existing literature (e.g., [6], [7], [8], [9]) only achieve the asymptotic stability, which implies that the consensus is reached as time tends to infinity. In practice, finite-time convergence is more preferable due to its tracking accuracy and better disturbance rejection [10], which therefore triggers an intensive research on finite-time cooperation control. Till now, most reported work in this community mainly addresses the finite-time consensus/synchronization for first- or second-order subsystems in a group [11], [12], [13], [14], [15], [16]. However, the convergence time of finite-time control design grows unboundedly along with the deviation of initial conditions from the equilibrium. As an extension of the finite-time stability, fixed-time stability is first discovered in [17] and then defined in [18] which assumes that the settling time is uniformly bounded and independent of initial conditions. In [18], a Lyapunov sufficient condition for fixed-time stability is given and an explicit estimated bound for the finite settling time is derived. The fixed-time stability exhibits an elegant property in some applications, like hybrid systems design. The first attempt in fixed-time consensus for integrator-type multi-agents can be found in [19], [20]. Then, the fixed-time consensus design framework is

This work was supported by the National Natural Science Foundation of China (No.61673034).

Z. Zuo is with the Seventh Research Division, and Science and Technology on Aircraft Control Laboratory, Beihang University (BUAA), Beijing 100191, China. (zzybobby@buaa.edu.cn)

B. Tian is with School of Electric and Automation Engineering, Tianjin University, Tianjin 300072, China. (bailing_tian@tju.edu.cn)

M. Defoort is with LAMIH, CNRS UMR 8201, University of Valenciennes and Hainaut-Cambrésis, Le Mont Houy, 59313 Valenciennes Cedex 9, France. (michael.defoort@univ-valenciennes.fr)

Z. Ding is with Control Systems Centre, School of Electrical and Electronic Engineering, University of Manchester, Sackville Street Building, Manchester M13 9PL, UK. (zhengtao.ding@manchester.ac.uk) generalized in [21] for the first-order integrator multi-agent systems subject to external disturbances. In view of the control singularity encountered for higher-order systems, the work [22] proposes a nonsingular fixed-time leader-follower consensus design for second-order multi-agent systems for the first time. In the very recent work [23], the authors improves the results in [22] and gives an estimate for the bound of the settling time, which is independent of initial conditions. Although several results on finite-time consensus for high-order systems, e.g., [24] and [25], have been reported, few results are reported on the fixed-time coordination control for high-order multiagent systems. Even the results in [16], [25], [22], [26] require each follower to obtain the inputs of its neighbors simultaneous, which causes a communication loop problem. It is nontrivial to extend the existing results for the first- or second-order case to higher-order one in a straightforward way. The difficulties arise from the establishment of unified Lyapunov functions for fixed-time stability and the control singularity encountered by a direct extension of existing methods, which implies that the corresponding fixed-time consensus design is quite challenging. Moreover, it is known that many systems in nature and engineering have high-order dynamics. Thus, it is imperative to study this issue for high-order multi-agent systems. Inspired by the work in [27] and our latest result in [28], a new cascade fixed-time consensus tracking design is proposed. The key feature of this paper is twofold: i) a new cascade control framework is proposed based on a fixed-time distributed observer to avoid the communication loop problem encountered by the decoupling design in [16], [22], [26]; ii) different from the finite-time (resp. fixed-time) stability achieved in [27] (resp. [28]) for a single system, the fixed-time stability can be guaranteed by the proposed design for multi-agent system with arbitrary order integrator dynamics without resorting to establishing involved Lyapunov functions.

The remainder of this paper is organized as follows. Some useful definitions and lemmas are recalled and then the fixed-time consensus tracking problem to be solved is formulated in Section II. In Section III, the cascade fixed-time consensus control framework with a fixed-time distributed observer is presented and the fixed-time stability analysis is carried out by using the Lyapunov technique. An illustrative simulation example with results are included in Section IV. Finally, concluding remarks are presented in Section V.

\section{Preliminaries and Problem Statement}

Notations: For any non-negative real number $\alpha$, the function $x \mapsto\lceil x\rfloor^{\alpha}$ is defined as $\lceil x\rfloor^{\alpha}=|x|^{\alpha} \operatorname{sign}(x)$ for any $x \in \mathbb{R}$. In terms of the definition, we have $x\lceil x\rfloor^{\alpha}=$ $|x|^{\alpha+1}$. For any $x=\left[x_{1}, x_{2}, \ldots, x_{N}\right]^{T} \in \mathbb{R}^{N}$, we define $\lceil x\rfloor^{\alpha}=\left[\operatorname{sign}\left(x_{1}\right)\left|x_{1}\right|^{\alpha}, \operatorname{sign}\left(x_{2}\right)\left|x_{2}\right|^{\alpha}, \ldots, \operatorname{sign}\left(x_{N}\right)\left|x_{N}\right|^{\alpha}\right]^{T}$. $\|x\|_{1}$ and $\|x\|_{2}$ denote 1 - and 2-norm of vector $x$, respectively. Throughout the paper, 1 denotes the vector with all elements one.

\section{A. Definitions and Lemmas}

Consider the system of differential equations

$$
\dot{x}(t)=f(t, x), \quad x(0)=x_{0},
$$


where $x \in \mathbb{R}^{n}$ and $f: \mathbb{R}_{+} \times \mathbb{R}^{n} \rightarrow \mathbb{R}^{n}$ is a nonlinear function. The solutions of (1) are understood in the sense of Filippov [29] if $f(t, x)$ is discontinuous. Suppose the origin is an equilibrium point of (1).

Definition 1: [10] The origin of the system (1) is said to be globally finite-time stable if it is globally asymptotically stable and any solution $X\left(t, x_{0}\right)$ of (1) reaches the origin at some finite moment, i.e., $X\left(t, x_{0}\right)=0, t \geq T\left(x_{0}\right)$, where $T: \mathbb{R}^{n} \rightarrow \mathbb{R}_{+} \cup 0$ is the settling time function.

Definition 2: [18] The origin of the system (1) is said to be globally fixed-time stable if it is globally uniformly finite-time stable and the settling time function $T$ is globally bounded, i.e., there exists a finite constant $T_{\max } \in \mathbb{R}_{+}$such that $T \leq T_{\max }$ and $x(t)=0$ for all $t \geq T$ and $x_{0} \in \mathbb{R}^{n}$.

Lemma 1: [18], [19] If there exists a continuous radially unbounded and positive definite function $V(x)$ such that

$$
\dot{V}(x) \leq-\alpha V^{p}-\beta V^{q}
$$

for some $\alpha, \beta>0, p>1,0<q<1$, then the origin of this system (1) is globally fixed-time stable and the settling time function $T$ can be estimated by

$$
T \leq T_{\max }:=\frac{1}{\alpha(p-1)}+\frac{1}{\beta(1-q)} .
$$

Furthermore, if $p=1+\frac{1}{\mu}$ and $q=1-\frac{1}{\mu}$ with $\mu>1$ are selected, the settling time function $T$ can be estimated by a less conservative bound

$$
T_{\max }:=\frac{\pi \mu}{2 \sqrt{\alpha \beta}} .
$$

Lemma 2: [28] Consider the $n$ th-order $(n \geq 2)$ integrator system

$$
\begin{aligned}
\dot{x}_{1}(t) & =x_{2}(t) \\
\dot{x}_{2}(t) & =x_{3}(t) \\
\vdots & \\
\dot{x}_{n}(t) & =u(t), \quad x(0)=x_{0}
\end{aligned}
$$

where $x=\left[x_{1}, x_{2}, \ldots, x_{n}\right]^{T} \in \mathbb{R}^{n}$ is the state vector and $u \in \mathbb{R}$ is the control input. Let the positive constants $k_{i}>0,(i=1,2, \ldots, n)$ be such that both the polynomial $s^{n}+k_{n} s^{n-1}+\cdots+k_{2} s+k_{1}$ and $s^{n}+3 k_{n} s^{n-1}+\cdots+3 k_{2} s+3 k_{1}$ are Hurwitz in terms of the Laplace operator $s$. There exists a constant $\epsilon \in\left(\frac{n-2}{n-1}, 1\right)$ such that, for every $\varrho \in(\epsilon, 1)$, the integrator system (5) can be stabilized at the origin in a fixed-time under the feedback control

$$
u(x)=-\sum_{i=1}^{n} k_{i}\left(\left\lceil x_{i}\right\rfloor^{\varrho_{i}}+\left\lceil x_{i}\right\rfloor+\left\lceil x_{i}\right\rfloor^{\varrho_{i}^{\prime}}\right)
$$

with parameters $\varrho_{i}$ and $\varrho_{i}^{\prime}$ satisfying, for $j=0,1, \ldots, n-1$,

$$
\varrho_{n-j}=\frac{\varrho}{(j+1)-j \varrho} \quad \text { and } \quad \varrho_{n-j}^{\prime}=\frac{2-\varrho}{j \varrho-(j-1)} .
$$

The proof of Lemma 2 in [28] is based on the bi-limit homogeneity [30]. Similarly, the proof could be also derived by following the same line as the proof of Theorem 2 given in the recent work [31]. Lemma 3: [22] Let $\xi_{1}, \xi_{2}, \ldots, \xi_{N} \geq 0$ and $0<p \leq 1$. Then,

$$
\sum_{i=1}^{N} \xi_{i}^{p} \geq\left(\sum_{i=1}^{N} \xi_{i}\right)^{p}
$$

Lemma 4: [22] Let $\xi_{1}, \xi_{2}, \ldots, \xi_{N} \geq 0$ and $p>1$. Then

$$
\sum_{i=1}^{N} \xi_{i}^{p} \geq N^{1-p}\left(\sum_{i=1}^{N} \xi_{i}\right)^{p}
$$

\section{B. Problem Formulation}

Consider $N+1$ agents with one leader and $N$ followers labeled by 0 and $i=1,2, \ldots, N$, respectively. The dynamics of the leader are described as follows

$$
\begin{aligned}
\dot{x}_{0,1}(t) & =x_{0,2}(t), \\
\dot{x}_{0,2}(t) & =x_{0,3}(t), \\
\vdots & \\
\dot{x}_{0, n}(t) & =u_{0}(t),
\end{aligned}
$$

where $x_{0}=\left[x_{0,1}, x_{0,2}, \ldots, x_{0, n}\right]^{T} \in \mathbb{R}^{n}$ is the state vector and $u_{0} \in \mathbb{R}$ is the control input of the leader. The dynamics of the $i$ th follower agent are described by [25]

$$
\begin{aligned}
\dot{x}_{i, 1}(t) & =x_{i, 2}(t), \\
\dot{x}_{i, 2}(t) & =x_{i, 3}(t), \\
\vdots & \\
\dot{x}_{i, n}(t) & =u_{i}(t)+\Delta_{i}(t),
\end{aligned}
$$

where $x_{i}=\left[x_{i, 1}, x_{i, 2}, \ldots, x_{i, n}\right]^{T} \in \mathbb{R}^{n}$ and $u_{i} \in \mathbb{R}$ represent the state vector and the control input, respectively, of the $i$ th agent, $\Delta_{i}$ represents the lumped uncertainties in the $i$ th agent. Note the term $\Delta_{i}$ may result from external disturbances, uncertain dynamics, or uncertainties in earlier states after differentiation.

The communication connection between the followers can be described by an edge set $\mathcal{E} \subseteq \mathcal{V} \times \mathcal{V}$ of a digraph $\mathcal{G}=\{\mathcal{V}, \mathcal{E}\}$, where $N$ followers are represented by a node set $\mathcal{V}=\{1,2, \ldots, N\}$. The adjacency matrix $A=\left[a_{i j}\right] \in \mathbb{R}^{N \times N}$ is defined by $a_{i j}>0$ if the follower $i$ can receive information from the follower $j$, otherwise $a_{i j}=0$. In the context, it is assumed that $a_{i i}=0$ (i.e., self loops are not allowed) and the topology is undirected. Denote by $D=\operatorname{diag}\left\{d_{1}, d_{2}, \ldots, d_{N}\right\}$ the degree diagonal matrix, where $d_{i}=\sum_{j=1}^{N} a_{i j}$ for $i=1,2, \ldots, N$. The graph Laplacian matrix is $L=\left[l_{i j}\right]=D-A$ with appropriate dimension. Let the diagonal matrix $B=\operatorname{diag}\left\{b_{1}, b_{2}, \ldots, b_{N}\right\}$ be the interconnection relationship between the leader and followers, where $b_{i}>0$ if the information of the leader is accessible by the $i$ th follower, otherwise $b_{i}=0$.

Several reasonable assumptions are made for the consensus tracking design discussed in this paper.

Assumption 1: Graph $\mathcal{G}$ is connected and at least one follower in graph $\mathcal{G}$ can get access to the states of the leader, i.e., $B \neq 0$.

Assumption 2: The input $u_{0}$ of the leader is unknown to any followers but its upper bound, denoted by $u_{0}^{\max }$, can be accessible by the $i$ th follower if $b_{i} \neq 0, i \in \mathcal{V}$.

Assumption 3: The unknown disturbance $\Delta_{i}(t)$ in (10) is uniformly bounded by a known constant $\delta$, i.e., $\left|\Delta_{i}(t)\right| \leq \delta$ for all $i \in \mathcal{V}$.

Remark 1: Assumption 1 is necessary for solving consensus tracking problem, and more discussion on this assumption can be referred to [32].

Remark 2: Assumption 2 is mild for physical systems since the input of the leader is known and its upper bound can be obtained a prior. Assumption 3 is made for robust control design and is conventional in sliding mode control [33].

The control objective is to design a distributed protocol $u_{i}$ using only relative information for each follower subject to external disturbances such that the fixed-time consensus is achieved, i.e., for $\forall x_{i}(0), \forall i \in$ $\mathcal{V}$, there exist a constant $T_{\max }$ such that

$$
\left\{\begin{array}{l}
\lim _{t \rightarrow T_{\max }}\left\|x_{i}(t)-x_{0}(t)\right\|_{2}=0 \\
x_{i}(t)=x_{0}(t), \quad \forall t>T_{\max } .
\end{array}\right.
$$




\section{FIXED-TIME CONSENSUS CONTROL PROTOCOL}

In this section, a new fixed-time consensus tracking protocol, based on a cascaded structure, is developed for multi-agent systems with high-order integrator dynamics.

\section{A. Distributed fixed-time observer}

In consideration that the information of the leader is available not to all followers but to only a portion of them, we may propose an observer for each follower to get an estimate of the state of the leader. Denote by $\hat{x}_{0, k}^{i}$ the estimate of the leader's state $x_{0, k}, k=1,2, \ldots, n$ for the $i$ th follower, $i \in \mathcal{V}$. A distributed fixed-time observer takes the following structure:

$$
\begin{aligned}
\dot{\hat{x}}_{0, k}^{i}= & \hat{x}_{0, k+1}^{i} \\
& +\alpha_{k} \operatorname{sign}\left(\sum_{j=1}^{N} a_{i j}\left(\hat{x}_{0, k}^{j}-\hat{x}_{0, k}^{i}\right)+b_{i}\left(x_{0, k}-\hat{x}_{0, k}^{i}\right)\right) \\
& +\beta_{k}\left[\sum_{j=1}^{N} a_{i j}\left(\hat{x}_{0, k}^{j}-\hat{x}_{0, k}^{i}\right)+\left.b_{i}\left(x_{0, k}-\hat{x}_{0, k}^{i}\right)\right|^{2},\right. \\
\dot{\hat{x}}_{0, n}^{i}= & \alpha_{n} \operatorname{sign}\left(\sum_{j=1}^{N} a_{i j}\left(\hat{x}_{0, n}^{j}-\hat{x}_{0, n}^{i}\right)+b_{i}\left(x_{0, n}-\hat{x}_{0, n}^{i}\right)\right) \\
& +\beta_{n}\left[\sum_{j=1}^{N} a_{i j}\left(\hat{x}_{0, n}^{j}-\hat{x}_{0, n}^{i}\right)+b_{i}\left(x_{0, n}-\hat{x}_{0, n}^{i}\right)\right]^{2} .
\end{aligned}
$$

Let the observation errors be

$$
\tilde{x}_{0, k}^{i}=\hat{x}_{0, k}^{i}-x_{0, k} .
$$

With (13), the observation error dynamics can be derived as

$$
\begin{aligned}
\dot{\tilde{x}}_{0, k}^{i}= & \tilde{x}_{0, k+1}^{i}+\alpha_{k} \operatorname{sign}\left(\sum_{j=1}^{N} a_{i j}\left(\tilde{x}_{0, k}^{j}-\tilde{x}_{0, k}^{i}\right)-b_{i} \tilde{x}_{0, k}^{i}\right) \\
& +\beta_{k}\left[\sum_{j=1}^{N} a_{i j}\left(\tilde{x}_{0, k}^{j}-\tilde{x}_{0, k}^{i}\right)-b_{i} \tilde{x}_{0, k}^{i}\right]^{2}, \\
\dot{\tilde{x}}_{0, n}^{i}= & \alpha_{n} \operatorname{sign}\left(\sum_{j=1}^{N} a_{i j}\left(\tilde{x}_{0, n}^{j}-\tilde{x}_{0, n}^{i}\right)-b_{i} \tilde{x}_{0, n}^{i}\right) \\
& +\beta_{n}\left[\sum_{j=1}^{N} a_{i j}\left(\tilde{x}_{0, n}^{j}-\tilde{x}_{0, n}^{i}\right)-b_{i} \tilde{x}_{0, n}^{i}\right]^{2}-u_{0} .
\end{aligned}
$$

Let $z_{i}=\left[\tilde{x}_{0, i}^{1}, \tilde{x}_{0, i}^{2}, \ldots, \tilde{x}_{0, i}^{N}\right]^{T}$ for $i=1,2, \ldots, n$. From (14), a compact form can be written as

$$
\begin{aligned}
\dot{z}_{k}= & z_{k+1}-\alpha_{k} \operatorname{sign}\left((L+B) z_{k}\right)-\beta_{k}\left\lceil(L+B) z_{k}\right\rfloor^{2}, \\
& (k=1,2, \ldots, n-1), \\
\dot{z}_{n}= & -\alpha_{n} \operatorname{sign}\left((L+B) z_{n}\right)-\beta_{n}\left\lceil(L+B) z_{n}\right\rfloor^{2} \\
& -\mathbf{1} u_{0} .
\end{aligned}
$$

The fixed-time convergence property of the estimation errors is summarized in the following theorem.

Theorem 1: If Assumptions 1 and 2 hold and the observer gains satisfy

$$
\begin{aligned}
\beta_{k} & =\frac{\varepsilon \sqrt{N}}{\left(2 \lambda_{\min }(L+B)\right)^{\frac{3}{2}}}, \quad \forall k=1,2, \ldots, n, \\
\alpha_{k} & =\varepsilon \sqrt{\frac{\lambda_{\max }(L+B)}{2 \lambda_{\min }(L+B)}}, \quad \forall k=1,2, \ldots, n-1, \\
\alpha_{n} & =u_{0}^{\max }+\varepsilon \sqrt{\frac{\lambda_{\max }(L+B)}{2 \lambda_{\min }(L+B)}},
\end{aligned}
$$

where $\varepsilon>0$, then the distributed observer (12) achieves the convergence of the observation errors to zero in a finite time which is bounded by

$$
T_{o}:=\frac{n \pi}{\varepsilon} .
$$

Proof: The proof consists of two steps. First, we show that the observation errors will not escape to infinity in finite time, i.e., the observation errors are bounded at any time interval $[0, t]$. Then, the fixed-time stability of (15) will be proved in a recursive manner. (i) Consider the following function

$$
V=\sum_{i=1}^{n} V_{i}=\frac{1}{2} \sum_{i=1}^{n} z_{i}^{T}(L+B) z_{i}
$$

where $V_{i}=\frac{1}{2} z_{i}^{T}(L+B) z_{i}, i=1,2, \ldots, n$, which are positive definite under Assumption 1 [32]. Differentiate $V$ along (15), and one obtain

$$
\begin{aligned}
\dot{V}= & \sum_{i=1}^{n-1} z_{i}^{T}(L+B) z_{i+1}-z_{n}^{T}(L+B) \mathbf{1} u_{0} \\
& -\sum_{i=1}^{n} \alpha_{i} z_{i}^{T}(L+B) \operatorname{sign}\left((L+B) z_{i}\right) \\
& -\sum_{i=1}^{n} \beta_{i} z_{i}^{T}(L+B)\left\lceil(L+B) z_{i}\right\rfloor^{2} \\
\leq & \frac{1}{2} \sum_{i=1}^{n-1} z_{i}^{T}(L+B) z_{i}+\frac{1}{2} \sum_{i=1}^{n-1} z_{i+1}^{T}(L+B) z_{i+1} \\
& -\sum_{i=1}^{n-1} \alpha_{i}\left\|(L+B) z_{i}\right\|_{1}-\sum_{i=1}^{n} \beta_{i}\left\|(L+B) z_{i}\right\|_{2} \\
& -\left(\alpha_{n}-u_{0}^{\max }\right)\left\|(L+B) z_{n}\right\|_{1} \\
\leq & 2 V
\end{aligned}
$$

where inequality $2 a^{T} b \leq a^{T} a+b^{T} b$ with vectors $a$ and $b$ is used to obtain the second inequality. Thus, $V$ is bounded at any time interval $[0, t]$, which implies from (20) that all $z_{i}$ will not escape to infinity in finite time. Moreover, it follows from (13) that all states of the observer (12) are bounded at any finite time interval if the state $x_{0}$ of the leader in (9) will not escape to infinity in finite time.

(ii) Consider the following Lyapunov function

$$
V_{n}=\frac{1}{2} z_{n}^{T}(L+B) z_{n}
$$

Its time derivative results in

$$
\begin{aligned}
\dot{V}_{n}= & z_{n}^{T}(L+B)\left(-\alpha_{n} \operatorname{sign}\left((L+B) z_{n}\right)-\beta_{n}\left\lceil(L+B) z_{n}\right\rfloor^{2}\right) \\
& -z_{n}^{T}(L+B) \mathbf{1} u_{0} \\
\leq & -\left(\alpha_{n}-u_{0}^{\max }\right)\left\|(L+B) z_{n}\right\|_{1} \\
& -\beta_{n} N^{-\frac{1}{2}}\left(2 \lambda_{\min }(L+B)\right)^{\frac{3}{2}} V_{n}^{\frac{3}{2}} \\
\leq & -\varepsilon V_{n}^{\frac{1}{2}}-\varepsilon V_{n}^{\frac{3}{2}} .
\end{aligned}
$$

Hence, Lemma 1 guarantees that $z_{n}$ is fixed-time stable at the origin with the settling time bounded by $T_{1}=\frac{\pi}{\varepsilon}$. 
After the convergence of $z_{n}$, the dynamics of $z_{n-1}$ reduce to

$$
\dot{z}_{n-1}=-\alpha_{n-1} \operatorname{sign}\left((L+B) z_{n-1}\right)-\beta_{n-1}\left\lceil(L+B) z_{n-1}\right\rfloor^{2} .
$$

Similar, we have that $z_{n-1}$ converges to zero in a fixed-time bounded by $T_{2}=2 T_{1}$. Recursively, we have that $z_{1}$ converges to zero within a fixed time horizon bounded by $T_{o}:=T_{n}=n T_{1}$. This completes the proof.

Remark 3: It is worth noting that the classical observer (e.g., the ones in [34], [35], [36] for consensus problem) is used to reconstruct the state of a system based on incomplete measurements. Different from the concept of conventional observer, the observer (12) is proposed to reconstruct the leader state in a distributed manner for each followers since only a portion of followers in a group can get access to the leader state. Theorem 1 guarantees that this observer could recover the leader state within a fixed time if Assumption 1 holds. Thus, the dynamic system (12) is still referred to as an "observer" without much confusion. Furthermore, it should be highlighted that if $x_{0, k}$ is transmitted from agent $i$ to agent $j$, instead of $\hat{x}_{0, k}^{i}$, the scheme becomes a centralized one. Then, problems of security and robustness to agent and communication link failures clearly make this strategy prohibitive. Hence, in this paper, the online reconstruction of the leader state for all followers is proposed to avoid such problems since only local information is transmitted.

Remark 4: The conclusion in Theorem 1 implies that $\hat{x}_{0, k}^{i}=x_{0, k}$ for all $t \geq T_{o}, k=1,2, \ldots, n, i \in \mathcal{V}$. In other words, each follower is able to accurately estimate the state of the leader after a period of time and thus we can use $\hat{x}_{0, k}^{i}$ in the protocol design based on a cascaded structure.

Remark 5: The settling time estimate (19) derived in Theorem 1 is independent of initial observation errors. To be specific, for any given desired $T_{o}, \varepsilon$ will be specified by (19) accordingly if the order of the multi-agent system is fixed, but the observer parameters (16) (18) will be then tuned according to the prescribed $\varepsilon$, the network topology and the number of agents in a group. In other words, (19) provides an explicit algorithm for adjusting the observer parameters to predefine the settling time estimate.

\section{B. Consensus Tracking Control}

Define the tracking error

$$
e_{i, k}=x_{i, k}-\hat{x}_{0, k}^{i}=x_{i, k}-x_{0, k}-\tilde{x}_{0, k}^{i},
$$

where $\tilde{x}_{0, k}^{i}$ is defined in (13), $k=1,2, \ldots, n$ and $i \in \mathcal{V}$. With (14), the time derivative of the tracking error $e_{i, k}$ can be derived as

$$
\begin{aligned}
\dot{e}_{i, k}= & e_{i, k+1}-\alpha_{k} \operatorname{sign}\left(\sum_{j=1}^{N} a_{i j}\left(\tilde{x}_{0, k}^{j}-\tilde{x}_{0, k}^{i}\right)-b_{i} \tilde{x}_{0, k}^{i}\right) \\
& -\beta_{k}\left[\sum_{j=1}^{N} a_{i j}\left(\tilde{x}_{0, k}^{j}-\tilde{x}_{0, k}^{i}\right)-b_{i} \tilde{x}_{0, k}^{i}\right]^{2}, \\
\dot{e}_{i, n}= & u_{i}+\Delta_{i}-\alpha_{n} \operatorname{sign}\left(\sum_{j=1}^{N} a_{i j}\left(\tilde{x}_{0, n}^{j}-\tilde{x}_{0, n}^{i}\right)-b_{i} \tilde{x}_{0, n}^{i}\right) \\
& -\beta_{n}\left[\sum_{j=1}^{N} a_{i j}\left(\tilde{x}_{0, n}^{j}-\tilde{x}_{0, n}^{i}\right)-b_{i} \tilde{x}_{0, n}^{i}\right]^{2} .
\end{aligned}
$$

By Theorem 1, we have $\tilde{x}_{0, k}^{i}=0$ for all $t \geq T_{o}$. Let $e_{k}=$ $\left[e_{1, k}, e_{2, k}, \ldots, e_{N, k}\right]^{T}$. From (9) and (10), for all $t \geq T_{o}$, the tracking error dynamics can be written in a compact form:

$$
\begin{aligned}
\dot{e}_{1}(t) & =e_{2}(t), \\
\dot{e}_{2}(t) & =e_{3}(t), \\
\vdots & \\
\dot{e}_{n}(t) & =U(t)+\Delta(t),
\end{aligned}
$$

where $U=\left[u_{1}, u_{2}, \ldots, u_{N}\right]^{T}, \Delta=\left[\Delta_{1}, \Delta_{2}, \ldots, \Delta_{N}\right]^{T}$.

To solve the fixed-time consensus tracking problem, we propose the following integral sliding surface for each follower:

$$
\begin{gathered}
s_{i}(t)=e_{i, n}(t)+\sum_{j=1}^{n} k_{i} \int_{0}^{t}\left(\left\lceil e_{i, j}(\tau)\right\rfloor^{\varrho_{i}}+\left\lceil e_{i, j}(\tau)\right\rfloor\right. \\
\left.+\left\lceil e_{i, j}(\tau)\right\rfloor^{\varrho_{i}^{\prime}}\right) \mathrm{d} \tau
\end{gathered}
$$

where $i \in \mathcal{V}$, the parameters $k_{i}, \varrho_{i}$ and $\varrho_{i}^{\prime}$ are selected as given in Lemma 2. Differentiating $s_{i}$ against time yields

$$
\begin{aligned}
\dot{s}_{i}(t)= & u_{i}(t)+\sum_{j=1}^{n} k_{i}\left(\left\lceil e_{i, j}(t)\right\rfloor^{\varrho_{i}}+\left\lceil e_{i, j}(t)\right\rfloor+\left\lceil e_{i, j}(t)\right\rfloor^{\varrho_{i}^{\prime}}\right) \\
& +\Delta_{i}(t)-\alpha_{n} \operatorname{sign}\left(\sum_{j=1}^{N} a_{i j}\left(\tilde{x}_{0, n}^{j}-\tilde{x}_{0, n}^{i}\right)-b_{i} \tilde{x}_{0, n}^{i}\right) \\
& -\beta_{n}\left\lceil\sum_{j=1}^{N} a_{i j}\left(\tilde{x}_{0, n}^{j}-\tilde{x}_{0, n}^{i}\right)-b_{i} \tilde{x}_{0, n}^{i}\right\rfloor^{2},
\end{aligned}
$$

and, for all $t \geq T_{o}$, dynamics $\dot{s}_{i}$ in (28) reduce to

$$
\begin{aligned}
\dot{s}_{i}(t)= & u_{i}(t)+\sum_{j=1}^{n} k_{i}\left(\left\lceil e_{i, j}(t)\right\rfloor^{\varrho_{i}}+\left\lceil e_{i, j}(t)\right\rfloor+\left\lceil e_{i, j}(t)\right\rfloor^{\varrho_{i}^{\prime}}\right) \\
& +\Delta_{i}(t) .
\end{aligned}
$$

In view of (29), a new fixed-time consensus tracking protocol can be prescribed as

$$
\begin{aligned}
u_{i}= & -\sum_{j=1}^{n} k_{i}\left(\left\lceil e_{i, j}\right\rfloor^{\varrho_{i}}+\left\lceil e_{i, j}\right\rfloor+\left\lceil e_{i, j}\right\rfloor^{\varrho_{i}^{\prime}}\right)-\rho \operatorname{sign}\left(s_{i}\right) \\
& -k_{i}\left(\left\lceil s_{i}\right\rfloor^{1+\frac{1}{\mu}}+\left\lceil s_{i}\right\rfloor+\left\lceil s_{i}\right\rfloor^{1-\frac{1}{\mu}}\right)
\end{aligned}
$$

with $\mu>1$ and $\rho \geq \delta$.

Remark 6: It is worth mentioning that the integral sliding surface (27) is invariant and insensitive to the observation errors $\tilde{x}_{0, k}^{i}$ if $\rho \geq$ $\delta$ in protocol (30) is large enough. However, a larger $\rho$ will result in a larger dithering. Thanks to the fixed-time convergence of (15), $\rho=\delta$ may be chosen for counteracting the unknown disturbances $\Delta_{i}$ merely.

The main result in this section is summarized in the following theorem.

Theorem 2: Consider multi-agent system (9) and (10). If Assumptions 1-3 hold, the fixed-time consensus tracking problem can be solved by the distributed protocol (30) with the decentralized observer (12).

Proof: The proof consists of three steps. We first show that all tracking errors $e_{i, k}, i \in \mathcal{V}, k=1,2, \ldots, n$, are bounded at any time interval $[0, t]$, i.e., no finite time escape occurs. Then, we show that the reaching phase can be finished in a fixed-time under the protocol (30). Finally, we will prove that the tracking errors $e_{i, k}$ defined in (24) converge to zero in a fixed-time during the sliding motion $s_{i}=0$. (i) Consider the following candidate Lyapunov function

$$
V=\sum_{i=1}^{N} V_{i}=\sum_{i=1}^{N}\left|s_{i}\right| .
$$


where $V_{i}=\left|s_{i}\right|$. Note that the definition of $\dot{V}$ becomes nontrivial when $s_{i}=0$, since the right hand side of (31) becomes discontinuous. Then, the concept of Filippov solutions and set-valued Lie derivative needs to be applied [29]. Define two sets $\mathcal{S}_{0}=\left\{s_{i}=0\right\}$ and $\mathcal{S}_{1}=$ $\left\{s_{i} \neq 0\right\}$, and then we have

$$
\left.\dot{V}_{i} \in \operatorname{sign}\left(s_{i}\right) \dot{s}_{i}\right|_{s_{i} \in \mathcal{S}_{0}}+\left.\operatorname{sign}\left(s_{i}\right) \dot{s}_{i}\right|_{s_{i} \in \mathcal{S}_{1}}
$$

where

$$
\operatorname{sign}(s)= \begin{cases}1 & s>0, \\ {[-1,1]} & s=0, \\ -1 & s<0 .\end{cases}
$$

In the sense of Filippov, the case in which $s_{i}=0$ holds for isolated time instants with zero measure can be disregarded in these time instants. If $s_{i}=0$ holds along an interval of time with positive measure, then $\dot{s}_{i}=0$ holds at these time instants. Thus, the time derivative of $V_{i}$ can be evaluated as $\dot{V}_{i}=\operatorname{sign}\left(s_{i}\right) \dot{s}_{i}$. Then, we have

$$
\dot{V}=\sum_{i=1}^{N} \operatorname{sign}\left(s_{i}\right) \dot{s}_{i}
$$

Taking into account (28) with (30), we have

$$
\begin{aligned}
\dot{V}= & \sum_{i=1}^{N} \operatorname{sign}\left(s_{i}\right)\left(\dot{e}_{i, n}\right. \\
& \left.\quad+\sum_{j=1}^{n} k_{i}\left(\left\lceil e_{i, j}\right\rfloor^{\varrho}+\left\lceil e_{i, j}\right\rfloor+\left\lceil e_{i, j}\right\rfloor_{i}^{\varrho_{i}^{\prime}}\right)\right) \\
= & \sum_{i=1}^{N} k_{i}\left(-\left|s_{i}\right|^{1+\frac{1}{\mu}}-\left|s_{i}\right|-\left|s_{i}\right|^{1-\frac{1}{\mu}}\right) \\
& +\sum_{i=1}^{N} \operatorname{sign}\left(s_{i}\right)\left(\Delta_{i}-\rho \operatorname{sign}\left(s_{i}\right)\right) \\
& -\alpha_{n} \sum_{i=1}^{N} \operatorname{sign}\left(s_{i}\right) \operatorname{sign}\left(\sum_{j=1}^{N} a_{i j}\left(\tilde{x}_{0, n}^{j}-\tilde{x}_{0, n}^{i}\right)-b_{i} \tilde{x}_{0, n}^{i}\right) \\
& -\beta_{n} \sum_{i=1}^{N} \operatorname{sign}\left(s_{i}\right)\left[\sum_{j=1}^{N} a_{i j}\left(\tilde{x}_{0, n}^{j}-\tilde{x}_{0, n}^{i}\right)-\left.b_{i} \tilde{x}_{0, n}^{i}\right|^{2}\right. \\
\leq & -\sum_{i=1}^{N} k_{i}\left(\left|s_{i}\right|^{1+\frac{1}{\mu}}+\left|s_{i}\right|+\left|s_{i}\right|^{1-\frac{1}{\mu}}\right) \\
& -\alpha_{n} \sum_{i=1}^{N} \operatorname{sign}\left(s_{i}\right) \operatorname{sign}\left(\sum_{j=1}^{N} a_{i j}\left(\tilde{x}_{0, n}^{j}-\tilde{x}_{0, n}^{i}\right)-b_{i} \tilde{x}_{0, n}^{i}\right) \\
& -\beta_{n} \sum_{i=1}^{N} \operatorname{sign}\left(s_{i}\right)\left[\sum_{j=1}^{N} a_{i j}\left(\tilde{x}_{0, n}^{j}-\tilde{x}_{0, n}^{i}\right)-\left.b_{i} \tilde{x}_{0, n}^{i}\right|^{2} \cdot\right.
\end{aligned}
$$

Recalling Theorem 1, we have shown that the estimation errors $\tilde{x}_{0, k}^{i}$ are fixed-time stable at the origin, which implies that $\tilde{x}_{0, k}^{i}$ are bounded, i.e., $\left|\tilde{x}_{0, k}^{i}\right| \leq \sigma_{k}^{i} \leq \bar{\sigma}$ for some constant $\bar{\sigma}$. Then, there exists a constant $C$ such that

$$
\dot{V} \leq-\sum_{i=1}^{N} k_{i}\left|s_{i}\right|+C=-\underline{k} V+C,
$$

with $\underline{k}=\min \left\{k_{1}, k_{2}, \ldots, k_{N}\right\}$. It follows that $V$ is bounded at any time interval $[0, t]$, which, in turn, implies that $s_{i}$ are bounded at any time interval. From (25) and the boundedness of all $\tilde{x}_{0, k}^{i}$, the finite-time escape of $e_{i, k+1}$ implies that $e_{i, k}$ will escape to infinity in finite time and have the same sign with $e_{i, k+1}$ ultimately, $k=$ $1,2, \ldots, n-1, i \in \mathcal{V}$, which leads to the unboundedness of $s_{i}$. This makes a contradiction. To this end, we can conclude that all tracking errors $e_{i, k}$ are bounded at any time interval $[0, t]$, i.e., no finite-time escape occurs. (ii) For all $t \geq T_{o}$, the tracking error dynamics can be described by (26). Taking the derivative of $V$ in (31) and following the same line in (32) result in

$$
\begin{aligned}
\dot{V} & \leq-\sum_{i=1}^{N} k_{i}\left(\left|s_{i}\right|^{1+\frac{1}{\mu}}+\left|s_{i}\right|+\left|s_{i}\right|^{1-\frac{1}{\mu}}\right) \\
& \leq-\sum_{i=1}^{N} k_{i}\left(\left|s_{i}\right|^{1+\frac{1}{\mu}}+\left|s_{i}\right|^{1-\frac{1}{\mu}}\right) .
\end{aligned}
$$

It follows from Lemmas 3 and 4 that $\dot{V}$ in (34) satisfies

$$
\begin{aligned}
\dot{V} & \leq-N^{-\frac{1}{\mu}} \underline{k}\left(\sum_{i=1}^{N}\left|s_{i}\right|\right)^{1+\frac{1}{\mu}}-\underline{k}\left(\sum_{i=1}^{N}\left|s_{i}\right|\right)^{1-\frac{1}{\mu}} \\
& \leq-N^{-\frac{1}{\mu}} \underline{k} V^{1+\frac{1}{\mu}}-\underline{k} V^{1-\frac{1}{\mu}} .
\end{aligned}
$$

From Lemma 1, the system trajectory will reach the sliding surface $s_{i}=0$ in a finite settling time bounded by $T_{r} \leq \pi \mu N^{\frac{1}{2 \mu}} /(2 \underline{k})$.

(iii) During the sliding motion (i.e., $s_{i} \equiv 0$ ), we have $\dot{s}_{i}=0$ and the reduced closed-loop dynamics can be derived as, for $t \geq T_{o}+T_{r}$,

$$
\begin{aligned}
\dot{e}_{1}(t) & =e_{2}(t), \\
\dot{e}_{2}(t) & =e_{3}(t), \\
\vdots & \\
\dot{e}_{n}(t) & =-\sum_{i=1}^{n} k_{i}\left(\left\lceil e_{i}\right\rfloor^{\varrho_{i}}+\left\lceil e_{i}\right\rfloor+\left\lceil e_{i}\right\rfloor^{\varrho_{i}^{\prime}}\right) .
\end{aligned}
$$

It follows from Lemma 2 that the reduced dynamics (36) are fixedtime stable at the origin, i.e., there exists a constant $T_{s}$ independent of initial conditions such that $e_{i, k} \rightarrow 0$ for all $t \geq T_{o}+T_{r}+T_{s}$, $i \in \mathcal{V}$ and $k=1,2, \ldots, n$, where $T_{o}$ is defined in Theorem 1 . Since $e_{i, k} \rightarrow 0$ implies $x_{i, k} \rightarrow x_{0, k}$ when $\tilde{x}_{0, k}^{i}=0$, this completes the proof.

Remark 7: It is worth noting that the tracking protocol proposed in the previous works [16], [22], [26] suffers from the communication loop problem due to the decoupling design, which prohibits the practical implementation. The proposed protocol (30), however, completely overcomes this drawback due to the introduction of a distributed fixed-time observer to decouple the information interaction between the follower and the leader.

Remark 8: In practical implementation, one may assign $\hat{x}_{0}^{i}=x_{0}$ in (24) and (30) for the follower who has a direct access to the leader state, i.e., the $i$ th follower for $b_{i} \neq 0$, to skip convergence phase of the observer, while the other followers with $b_{i}=0$ use the reconstructed state $\hat{x}_{i}$.

\section{Simulation}

In this section, an illustrative example is presented to show the efficacy of the proposed fixed-time consensus tracking protocol. The multi-agent system consists of one leader indexed by 0 and five followers indexed by 1 to 5 . The dynamics of the leader and the followers are described by, respectively,

$$
\begin{aligned}
& \dot{x}_{0,1}(t)=x_{0,2}(t), \\
& \dot{x}_{0,2}(t)=x_{0,3}(t), \\
& \dot{x}_{0,3}(t)=u_{0}(t),
\end{aligned}
$$

and

$$
\begin{aligned}
\dot{x}_{i, 1}(t) & =x_{i, 2}(t), \\
\dot{x}_{i, 2}(t) & =x_{i, 3}(t), \\
\dot{x}_{i, 3}(t) & =u_{i}(t)+\Delta_{i}(t), \quad(i=1,2, \ldots, 5)
\end{aligned}
$$


where $x_{k, 1}, x_{k, 2}, x_{k, 3}$ and $u_{k},(k=0,1,2, \ldots, 5)$, represent the position, the velocity, the acceleration and the input of the leader and the follower, respectively, $\Delta_{i}$ are the disturbances.

In simulation, the input of the leader is selected as $u_{0}=-\sin (0.5 t)$ and the external disturbances $\Delta_{i}=0.1 \sin \left(x_{i}\right)$ are used. The initial values of the leader are fixed as $x_{0}=[-8,0,2]^{T}$. The interconnection topology is illustrated in Fig.1 and the corresponding Laplacian matrix is

$$
L=\left[\begin{array}{ccccc}
3 & -1 & -1 & -1 & 0 \\
-1 & 2 & -1 & 0 & 0 \\
-1 & -1 & 2 & 0 & 0 \\
-1 & 0 & 0 & 2 & -1 \\
0 & 0 & 0 & -1 & 1
\end{array}\right]
$$

and the leader accessibility matrix is $B=\operatorname{diag}\{1,0,0,0,1\}$. The controller parameters $k_{1}=2, k_{2}=3, k_{3}=5$ and $\varrho=0.7$ are selected and it can be verified that the resulting polynomials $s^{3}+5 s^{2}+3 s+2$ and $s^{3}+15 s^{2}+9 s+6$ are both Hurwitz, i.e., their respective roots $\{-4.4241,-0.2880 \pm \mathrm{j} 0.6076\}$ and $\{-14.4041,-0.2980 \pm j 0.5725\}$ are all located in the left complex plane. The observer parameters $\alpha_{1}=\alpha_{2}=0.8, \alpha_{3}=2$ and $\beta_{1}=\beta_{2}=\beta_{3}=1.2$ are fixed in the simulation.

The simulation is carried out using Euler method with a fixed integration step equal to $10^{-4}$ [37]. In order to validate the efficacy of the proposed design, two scenarios for different initial values of the followers are considered:

$$
\text { (i) } \begin{aligned}
& x_{1}=[-10,0,0], x_{2}=[-5,0,0], x_{3}=[0,0,0], \\
& x_{4}=[5,0,0], x_{5}=[10,0,0] ; \\
& \text { (ii) } \quad x_{1}=[-100,0,0], x_{2}=[-50,0,0], x_{3}=[10,0,0], \\
& x_{4}=[50,0,0], x_{5}=[100,0,0] .
\end{aligned}
$$

The simulation results for the both scenarios are presented in Figs. 27. Figs. 2-4 show that the position, the velocity and the acceleration errors between each follower and the leader converge to zero very fast, which implies that the consensus tracking is achieved in a fixed time. From Fig. 6, we can observe that the chattering happens in the control inputs of followers, which results from the discontinuous term $\rho \operatorname{sign}\left(s_{i}\right)$ incorporated in the protocol (30). With a larger initial deviation of the followers and without re-tuning the parameters, Fig. 7 shows the convergence time is almost the same as that in Fig. 2 , which demonstrates a weak dependence of the settling time on initial conditions due to the fixed-time consensus design. In addition, note from Figs. 5 and 8 that the distributed consensus observer achieves almost the same convergence speed to the leader's state under different initial scenarios.

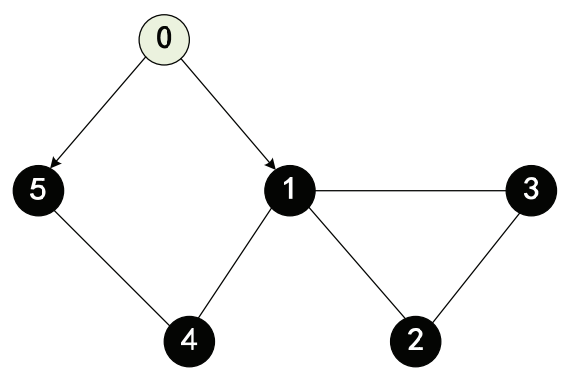

Fig. 1. Information flow among the leader and the followers

\section{Conclusions}

In this paper, the fixed-time consensus tracking problem for multiagent systems with high-order integrator dynamics is addressed.

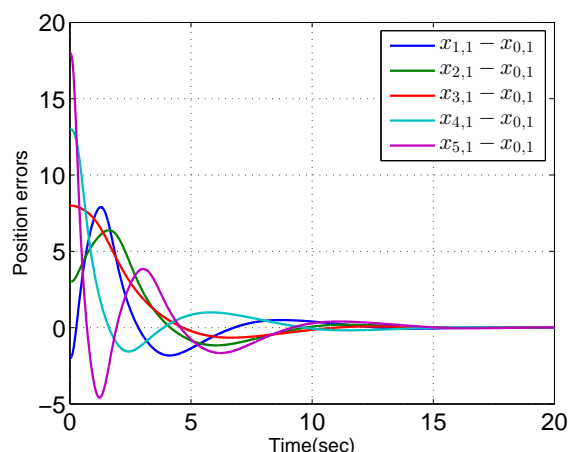

Fig. 2. Profiles of the position tracking errors: Scenario (i)

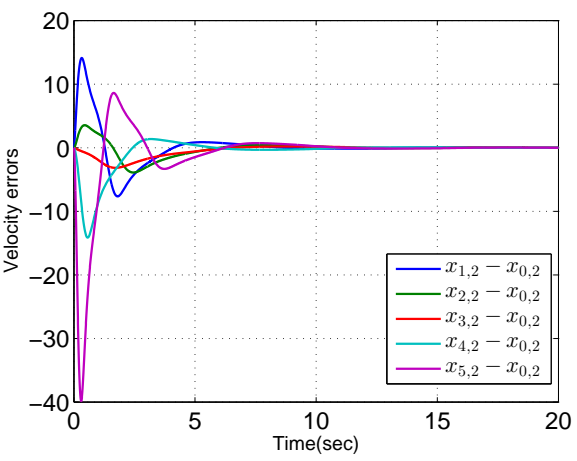

Fig. 3. Profiles of the velocity tracking errors: Scenario (i)

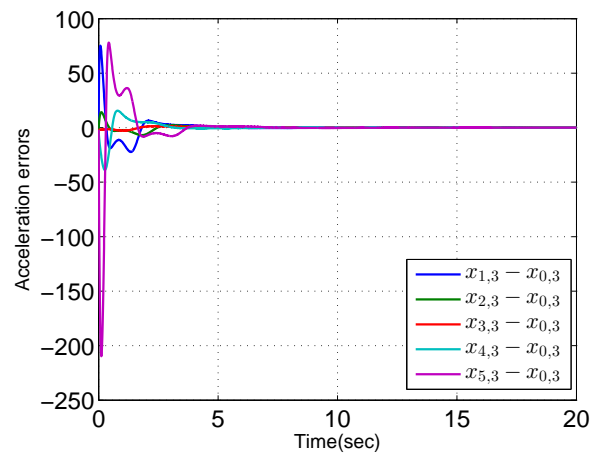

Fig. 4. Profiles of the acceleration tracking errors: Scenario (i)

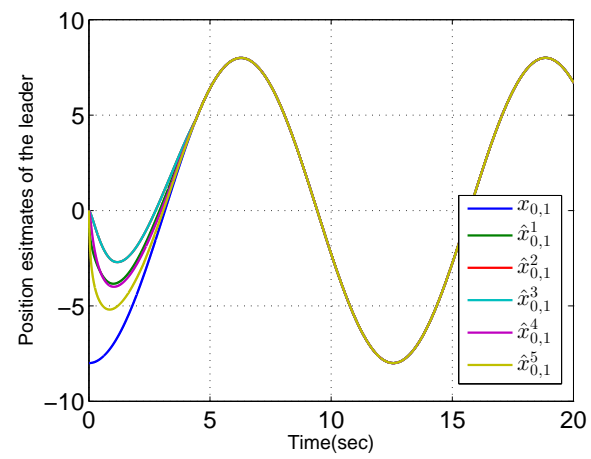

Fig. 5. Profiles of the estimates of the leader's position: Scenario (i)

A distributed observer based consensus tracking design framework is developed, which provides an effective way to construct the 


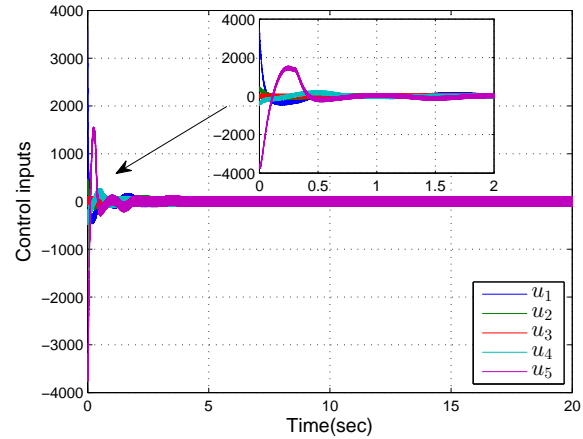

Fig. 6. Profiles of the control inputs of all followers: Scenario (i)

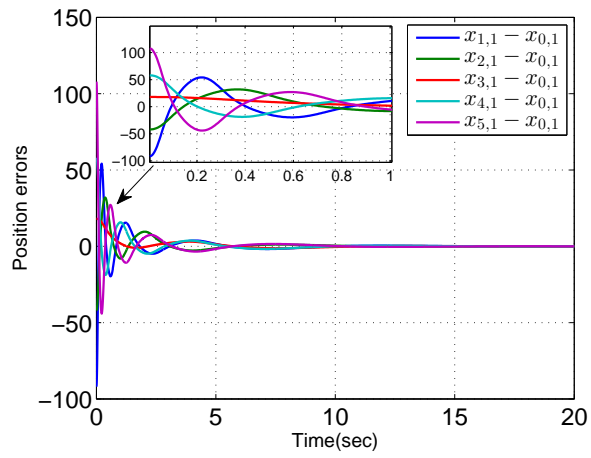

Fig. 7. Profiles of the position tracking errors: Scenario (ii)

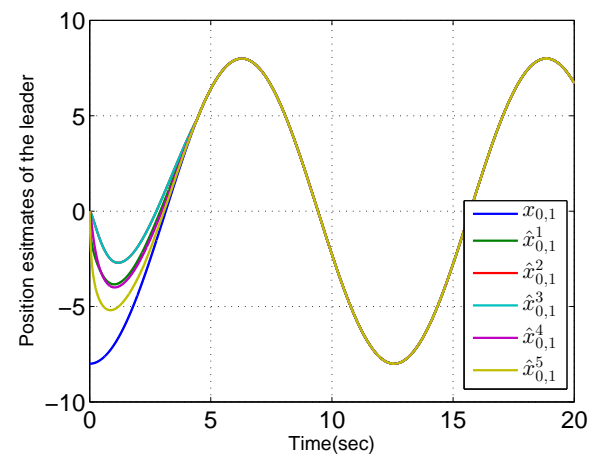

Fig. 8. Profiles of the estimates of the leader's position: Scenario (ii)

fixed-time consensus protocol for multi-agent systems with arbitrary order integrator dynamics. Future work includes the extension of the proposed framework to more general multi-agent systems with directed topology.

\section{REFERENCES}

[1] A. Karimoddini, H. Lin, B.-M. Chen, and T.-H. Lee, "Hybrid threedimensional formation control for unmanned helicopters," Automatica, vol. 49, no. 2, pp. 424-433, 2013.

[2] Z. Zuo, W. Yang, L. Tie, and D. Meng, "Fixed-time consensus for multiagent systems under directed and switching interaction topology," in American Control Conference (ACC), Portland, Oregon, USA, Jun 2014, pp. 5133-5138.

[3] X. Wu, Y. Tang, J. Cao, and W. Zhang, "Distributed consensus of stochastic delayed multi-agent systems under asynchronous switching," IEEE Transactions on Cybernetics, vol. 46, no. 8, pp. 1817-1827, 2016.

[4] R. Olfati-Saber and R. M. Murray, "Consensus problems in networks of agents with switching topology and time delays," IEEE Transaction on Automatic Control, vol. 49, no. 9, pp. 1520-1533, 2004.
[5] Y. Kim and M. Mesbahi, "On maximizing the second smallest eigenvalue of a state-dependent graph laplacian," IEEE Transaction on Automatic Control, vol. 51, no. 1, pp. 116-120, 2006.

[6] W. Ren and R. Beard, "Consensus seeking in multiagent systems under dynamically changing interaction topologies," IEEE Transaction on Automatic Control, vol. 50, no. 5, pp. 655-661, 2005.

[7] Z. Li, Z. Duan, G. Chen, and L. Huang, "Consensus of multi-agent systems and synchronization of complex networks: a unified viewpoint," IEEE Transactions Circuits and Systems-I: Regular Papers, vol. 57, no. 1, pp. 213-224, 2010.

[8] W. Yu, G. Chen, and M. Cao, "Some necessary and sufficient conditions for second-order consensus in multi-agent dynamical systems," Automatica, vol. 46, no. 6, pp. 1089-1095, 2010.

[9] Z. Ding, "Consensus disturbance rejection with disturbance observers," IEEE Transactions on Industrial Electronics, vol. 62, no. 9, pp. 58295837, 2015.

[10] S. Bhat and D. Bernstein, "Finite time stability of continuous autonomous systems," SIAM Journal of Control Optimization, vol. 38, no. 3, pp. 751-766, 2000.

[11] J. Cortés, "Finite-time convergent gradient flows with applications to network consensus," Automatica, vol. 42, no. 11, pp. 1993-2000, 2006.

[12] L. Wang and F. Xiong, "Finite-time consensus problems for networks of dynamic agents," IEEE Transactions Automatic Control, vol. 55, no. 4, pp. 950-955, 2010.

[13] Y. Cao and W. Ren, "Finite-time consensus for multi-agent networks with unknown inherent nonlinear dynamics," Automatica, vol. 50, no. 10, pp. 2648-2656, 2014.

[14] H. Du, S. Li, and C. Qian, "Finite-time attitude tracking control of spacecraft with application to attitude synchronization," IEEE Transactions Automatic Control, vol. 56, no. 11, pp. 2711-2717, 2011.

[15] J. Zhou, Q. Hu, and M. Friswell, "Decentralized finite time attitude synchronization control of satellite formation flying," Journal of Guidance Control and Dynamics, vol. 36, no. 1, pp. 185-195, 2013.

[16] S. Khoo, L. Xie, and Z. Man, "Robust finite-time consensus tracking algorithm for multirobot systems," IEEE/ASME Transactions on Mechatronics, vol. 14, no. 2, pp. 219-228, 2009.

[17] V. Andrieu, L. Praly, and A. Astolfi, "Homogeneous approximation, recursive observer design and output feedback," SIAM Journal on Control and Optimization, vol. 47, no. 4, p. 1814C1850, 2008.

[18] A. Polyakov, "Nonlinear feedback design for fixed-time stabilization of linear control systems," IEEE Transactions on Automatic Control, vol. 57, no. 8, pp. 2106-2110, 2012.

[19] S. Parsegov, A. Polyakov, and P. Shcherbakov, "Fixed-time consensus algorithm for multi-agent systems with integrator dynamics," in 4th IFAC Workshop on Distributed Estimation and Control in Networked Systems, Koblenz, Germany, Sept. 2013, pp. 110-115.

[20] Z. Zuo and L. Tie, "A new class of finite-time nonlinear consensus protocols for multi-agent systems," International Journal of Control, vol. 87, no. 2, pp. 363-370, 2014.

[21] _ _ "Distributed robust finite-time nonlinear consensus protocols for multi-agent systems," International Journal of Systems Science, vol. 47, no. 6, pp. 1366-1375, 2016.

[22] Z. Zuo, "Non-singular fixed-time consensus tracking for second-order multi-agent networks," Automatica, vol. 54, no. 4, pp. 305-309, 2015.

[23] J. Fu and J. Wang, "Fixed-time coordinated tracking for second-order multi-agent systems with bounded input uncertainties," Systems \& Control Letters, vol. 93.

[24] Y. Zhou, X. Yu, C. Sun, and W. Yu, "Higher order finite-time consensus protocol for heterogeneous multi-agent systems," International Journal of Control, vol. 88, no. 2, pp. 285-294, 2015.

[25] S. Khoo, L. Xie, S. Zhao, and Z. Man, "Multi-surface sliding control for fast finite-time leader-follower consensus with high order siso uncertain nonlinear agents," International Journal of Robust and Nonlinear Control, vol. 24, no. 6, pp. 2388-2404, 2014.

[26] H. Zhang and F. Lewis, "Adaptive cooperative tracking control of higherorder nonlinear systems with unknown dynamics," Automatca, vol. 48, no. 7, pp. 1432-1439, 2012.

[27] M. Defoort, T. Floquet, A. Kokosy, and W. Perruquetti, "A novel higher order sliding mode control scheme," Systems and Control Letters, vol. 58, no. 2, pp. 102-108, 2009.

[28] B. Tian, Z. Zuo, H. Wang, Y. Lin, and X. Liang, "A novel fixed-time control scheme for high order systems with both matched and mismatched disturbances," IET Control Theory and Applications, submitted.

[29] A. Filippov, Differential equations with discontinuous right-hand side. Kluwer Academic, Dordrecht, 1988. 
[30] V. Andrieu, L. Praly, and A. Astolfi, "Homogeneous approximation, recursive observer design and output feedback," SIAM Journal on Control and Optimization, vol. 47, no. 4, pp. 1814-1850, 2008.

[31] M. Basin, Y. Shtessel, and F. Aldukali, "Continous finite- and fixedtime high-order regulators," Journal of The Franklin Institute, vol. 253, no. 18, pp. 5001-5012, 2016.

[32] H. Zhang and F. Lewis, "Adaptive cooperative tracking control of higherorder nonlinear systems with unknown dynamics," Automatica, vol. 48, no. 7, pp. 1432-1439, 2012.

[33] V. Utkin, Sliding Modes in Control and Optimization. Springer Verlag, Berlin, 1992.

[34] A.-S.-M. Isira, Z. Zuo, and Z. Ding, "Leader-follower consensus control of lipschitz nonlinear systems by output feedback," International Journal of Systems Science, vol. 47, no. 16, pp. 3772-3781, 2016.

[35] Y. Cao, L. Zhang, C. Li, and M.-Z.-Q. Chen, "Observer-based consensus tracking of nonlinear agents in hybrid varying directed topology," IEEE Transactions Cybernetics, published online, doi:10.1109/TCYB.2016.2573138.

[36] J. C. W. X. X. Liu, D.-W.-C. Ho, "Discontinuous observers design for finite-time consensus of multiagent systems with external disturbances," IEEE Transactions Neural Network and Learning Systems, published online, doi:10.1109/TNNLS.2016.2599199.

[37] A. Levant, "On fixed and finte time stability in sliding mode control," in 52nd IEEE Conference on Decision and Control, Florence, Italy, Dec. 2013, pp. 4260-4265. 